\title{
Absolute Stability of Melvin's Magnetic Universe*
}

\author{
KIP S. THORNE $\dagger$ \\ Palmer Physical Laboratory, Princeton University, Princeton, New Jersey
}

(Received 9 November 1964)

\begin{abstract}
The physical structure of Melvin's magnetic universe is briefly examined and its dynamical behavior under arbitrarily large radial perturbations is discussed. It is shown that no radial perturbation can cause the magnetic field to undergo gravitational collapse to a singularity or electromagnetic explosion to infinite dispersion. Rather, when arbitrarily perturbed inside a finite region, the magnetic and gravitational fields undergo damped, turbulent oscillation until they have radiated away from the perturbed region all the energy associated with the perturbation. Then they settle down into Melvin's unperturbed, static configuration.
\end{abstract}

\section{INTRODUCTION AND SUMMARY}

A CYLINDRICAL electromagnetic universe (C.E.U.) is a system composed entirely of electromagnetic and gravitational fields, which exhibits whole-cylinder symmetry. (This means it is invariant under rotation about and translation along an axis of symmetry, and under reflection in planes containing that axis or perpendicular to it.) There has been much interest in cylindrical electromagnetic universes recently, ${ }^{1-3}$ partly because of a belief that certain C.E.U.'s might undergo gravitational collapse, ${ }^{4}$ and partly because a C.E.U. is the limiting case of a toroidal electromagnetic configuration (geon) ${ }^{5}$ when the major radius of the active region becomes much larger than its minor radius. ${ }^{4,6}$

The most general cylindrical electromagnetic universe has electric- and magnetic-field lines which lie in the cylindrical surfaces perpendicular to the radial direction; radial components would lead to singularities on the symmetry axis. Those C.E.U.'s with purely longitudinal magnetic field and purely azimuthal electric field have been the subject of particularly intensive investigation by Melvin., ${ }^{1,2} \mathrm{He}$ has shown that all static configurations of this type are related by a simple scale transformation which shortens radial distances, while increasing electromagnetic-field strengths. There is no electric field in these static configurations, and the magnetic field is concentrated near the axis of symmetry, dying out quite rapidly beyond a certain proper

\footnotetext{
* This work was supported in part by the U. S. Air Force Office of Scientific Research and by the National Science Foundation.

$\dagger$ Danforth Foundation Fellow, 1963-64; National Science Foundation Predoctoral Fellow 1964-65.

${ }^{1}$ M. A. Melvin, Phys. Letters 8, 65 (1964). Henceforth this paper will be referred to as Melvin I.

${ }_{2}^{2}$ M. A. Melvin, preceding paper, Phys. Rev. 139, B225 (1965). Henceforth this paper will be referred to as Melvin II.

${ }^{3}$ M. Misra and L. Radhakrishna, Proc. Natl. Inst. Sci. India 28A, 632 (1962); B. K. Harrison, Phys. Rev. 138, B488 (1965); K. P. Singh, L. Radhakrishna, and R. Sharan, Ann. Phys. (N. Y.) 32, 46 (1965)

${ }^{4} \mathrm{~J}$. A. Wheeler, in Relativity, Groups and Topology, edited by B. and C. DeWitt (Gordon and Breach Publishers, Inc., New York, 1964).

${ }^{5}$ For a discussion of geons see J. A. Wheeler, Geometrodynamics (Academic Press Inc., New York, 1962).

K. S. Thorne, in Quasistellar Sources and Gravitational Collapse, edited by I. Robinson, A. Schild, and E. Schücking (University of Chicago Press, Chicago, 1965).
}

radius $\bar{a}$. We will call a representative one of these static C.E.U.'s Melvin's magnetic universe; and we will call that value of $\bar{a}$ which singles it out the range radius of the universe.

Shortly after Melvin discovered his universe, as a solution to the Einstein-Maxwell equations of general relativity, Wheeler ${ }^{4}$ showed that it could also be obtained from Newton's theory of gravitation, and that its magnetic field configuration gives a maximum of the Newtonian energy per unit length, subject to the constraint of fixed magnetic flux. For this reason, he tentatively described Melvin's universe as a highly concentrated bundle of magnetic-field lines, so distributed that the gravitational attraction between field lines delicately balances their Maxwell-Faraday repulsion. If the configuration were squeezed slightly, gravitational forces would overwhelm electromagnetic forces, and the field lines would implode. Conversely, distending the configuration would cause explosion.

Subsequent analyses have contradicted this picture: Melvin II has found his universe to be stable against small radial perturbations; and, independently, the author has proved stability against arbitrarily large perturbations, which are confined to a finite region about the symmetry axis. (Cf. Sec. III.)

How can this extreme stability be understood in physical terms; and why did the Newtonian analysis, on which the belief of instability was based, give the wrong result? To answer these questions, we need a familiarity with the concept of "cylindrical energy" or " $C$ energy."

In a separate communication ${ }^{7}$ the author introduces the concept of $C$ energy for whole-cylinder-symmetric systems and shows that for these systems it plays a role in Einstein's theory analogous to that played in Newton's theory by the mass-energy (rest mass, plus internal energy, plus negative gravitational energy). In particular, $C$ energy has the following properties: (1) $C$ energy takes the form of a contravariant vector $P^{i}$, which obeys the conservation law $P_{\alpha i}^{i}=0$. (2) $C$ energy is localizable and locally measurable: The component of $P^{i}$ along the world line of an observer is the $C$-energy density he measures in a local Lorentz frame;

${ }^{7}$ K. S. Thorne, Phys. Rev. 138, B251 (1965). 
and the component of $P^{i}$ along a direction $u^{i}$ orthogonal to his world line is the $C$-energy flux he sees flowing in that direction. (3) The $C$ energy per unit of (grouptheoretically-defined) coordinate length, integrated over the interior of a solid cylinder, is equal to the cylinder's proper mass per unit length, in the limit of small mass; but just as the Newtonian potential of a cylinder diverges at infinity, so there is an infinite total $C$ energy per unit coordinate length-out to infinity-in the gravitational field of a cylinder. (4) The total $C$ energy of a thin-ring torus of small proper mass (Newtonian limit) is the same as its Schwarzschild mass. (5) $C$ energy is propagated by Einstein-Rosen gravitational waves and by cylindrical electromagnetic waves. (6) In vacuo, and in cylindrical electromagnetic universes, the $C$ energy per unit coordinate length, $E$, can be split into a kinetic term $K$ plus a potential term $P$ such that: $\delta \int_{t_{1}} t_{2}(P-K) d t=0$ is an action principle for the EinsteinMaxwell field equations; $K=0$ for static systems; and both $P$ and $E=P+K$ are absolutely minimized (subject to certain constraints) by static systems. In particular, denote by $\zeta$ the group-theoretically-defined longitudinal coordinate for a cylindrical electromagnetic universe, and by $g_{\zeta \zeta}(\rho) \delta \zeta$ the proper displacement at radius $\rho$ associated with the invariant translation $\zeta \rightarrow \zeta+\delta \zeta$ of the universe. Then, of all cylindrical electromagnetic universes, Melvin's magnetic universe gives the absolute minimum of the $C$ energy per unit coordinate length inside any cylinder about the symmetry axis, subject to the constraints of fixed magnetic flux through the cylinder and fixed ratio $g_{\zeta \zeta}$ (surface of cylinder) $/ g_{\zeta \zeta}$ (at axis of symmetry).

The minimum C-energy property of Melvin's magnetic universe is in sharp contrast with its maximum Newtonian-energy property. Which one is truly relevant to the stability problem? Because space-time is far from asymptotically flat around Melvin's universe, Newtonian theory is not a good approximation to Einstein's theory; we must abandon it and its associated energy in favor of the full Einstein theory and the $C$ energy concept. Nevertheless, it is remarkable that Newton's theory predicts Melvin's universe to be an equilibrium configuration (though an unstable one), despite the nonapplicability of that theory to wholecylinder-symmetric systems.

Because of its minimum $C$-energy property, Melvin's magnetic universe is the most diffuse configuration of electromagnetic and gravitational fields possible, subject to the constraints of fixed magnetic flux, and fixed ratio $g_{\zeta \zeta}(\infty) / g_{\zeta \zeta}(0)$. This diffuseness need not come as a complete surprise, for in his original paper ${ }^{1}$ Melvin remarked that in his universe "(the charge-current potential) $H^{i j}=(-g)^{1 / 2} f^{i j}$ is everywhere a constant .... It is only because of the nonuniformity of the metric that [the physical magnetic field] $B$, and also the stress-energy density, show a concentrated distribution." (Italics are Melvin's.) Melvin's remark provides an intuitive way of looking at his configuration: One can think of the magnetic field as "actually" spread out uniformly over all space, while its own stress-energy "warps space up around it" and makes it "appear" to be concentrated in a small region about the symmetry axis. The difference between the "actual" situation and the "apparent" situation is like the difference between the bare mass and renormalized mass of the electron. It is the "apparent" situation which an observer would see.

The minimum $C$-energy property of Melvin's magnetic universe is in complete agreement with its stability against perturbations, large and small. It also agrees with and provides the basis for the results discussed in Sec. III of this paper: If Melvin's configuration of magnetic and gravitational fields is arbitrarily perturbed inside some region about the symmetry axis and then released, the lines of force in the perturbed region will lash about turbulently, and radiation will flow away toward radial infinity carrying with it the excess $C$ energy associated with the initial perturbation. As the excess $C$ energy is radiated away, the field lines will gradually settle back down to the configuration of minimum $C$ energy, Melvin's static magnetic universe. No matter how extreme the initial perturbation, the magnetic-field lines of Melvin's universe cannot be forced to implode or explode, because both a completely collapsed and a completely dispersed configuration contain infinitely more $C$ energy than the unperturbed configuration. ${ }^{8}$

"Of what value is such a detailed analysis of a system which certainly does not exist anywhere in our universe?" it might be asked. The reply is fourfold:

(1) There is now reason to believe ${ }^{9}$ that very strong gravitational fields may play an important role in astrophysical processes-e.g., in supernovae, in neutrons stars, in quasistellar radio sources, and in the nuclei of some galaxies. Einstein's general theory of relativity is, at present, the most widely accepted theory of strong gravitational fields. However, amazingly little is known about the dynamical interaction between strong gravitational fields and their sources ("geometrodynamics") within the framework of Einstein's theory. Any geometrodynamical analysis, such as the one discussed here, is of great value in building up our knowledge of the types of processes allowed by Einstein's theory.

(2) Melvin's magnetic universe may be of particularly great value in understanding the nature of extra-

\footnotetext{
8 This corrects a statement made by the author at the Dallas Symposium on Gravitational Collapse, Dallas, 1963 (unpublished), to the effect that preliminary results of numerical calculations indicated that Melvin's universe was probably unstable against gravitational collapse. The final results of those calculations, which support the present view of Melvin's universe, are reported in Sec. III of this paper.

${ }^{9}$ Quasistellar Sources and Gravitational Collapse, edited by I. Robinson, A. Schild, and E. Schücking (University of Chicago Press, Chicago, 1965); F. Hoyle, W. A. Fowler, G. R. and E. M. Burbidge, Astrophys. J. 139, 909 (1964); H. Y. Chiu and E. E. Salpeter, Phys. Rev. Letters 12, 413 (1964).
} 
galactic sources of radio waves: Many strong radio sources take the form of two synchrotron-radiationemitting regions situated on opposite sides of a galaxy. The most popular theory to account for this is that the magnetic fields and high-energy particles responsible for the synchrotron radiation were blown out of the galactic nucleus in a giant explosion. Such an explosion would have to be highly directional in order to explain the observations. R. H. Dicke (private communication) has suggested that it could result from the gravitational collapse of a very prolate spheroid or of a cylinder whose axis of symmetry is defined by a strong magnetic field. Collapse perpendicular to the axis of symmetry would occur catastrophically, with a consequent ejection of material out of the ends. An important question to ask is: What effects would general relativity have on such a model? This question is most easily answered by studying "cylindrical model universes" such as Melvin's magnetic universe, which are idealizations of finite cylinders. The results described in this paper suggest that: (a) A strong magnetic field along the axis of symmetry may halt the cylindrical collapse of a finite cylinder before a singularity is reached. (b) Electromagnetic and gravitational waves will be profusely emitted by such a collapsing cylinder.

(3) Essentially nothing is known about the gravitational collapse of nonspherical bodies within the framework of Einstein's theory. A particular nonspherical body whose collapse could be studied is a toroidal magnetic geon ${ }^{4-6}$ consisting of a bundle of magneticfield lines in a toroidal configuration. Now that we understand the dynamics of Melvin's universe, we can investigate that toroidal geon, of which it is the idealization in the limit of infinitely large major radius. Will such a toroidal configuration be completely stable; like Melvin's universe? Probably not. It may be stable against collapse of its minor radius, but there are powerful arguments ${ }^{4,6}$ suggesting instability against collapse of its major radius.

(4) Just as magnetic fields play a major role in normal astrophysical processes (e.g., in radio galaxies, in galactic spiral arms, in the Crab nebula), so they may be important in strong-gravitational-field phenomena. A first step toward understanding the roles they might play there is the analysis of whole-cylindersymmetric configurations such as Melvin's universe. On the basis of the stability of Melvin's universe against gravitational collapse (proved in Sec. III), together with other examples of the resistance of magnetic and electric flux against compression into very small regions, the author has suggested elsewhere ${ }^{7,10}$ the principle of flux resistance to gravitational collapse: In any configuration of electromagnetic fields gravitationally collapsing to a singularity, the total electric and magnetic fluxes across every 2-surface through the collapsing region must

${ }^{10} \mathrm{~K}$. S. Thorne, in Proceedings of the Second Texas Symposium on Relativistic Astrophysics (University of Chicago Press, Chicago, 1965). vanish as the singularity is reached-a nonzero flux will stop the collapse.

The purpose of the remainder of this paper is twofold: (1) to present a brief discussion of the physical structure of Melvin's magnetic universe-including its magnetic field distribution, its $C$-energy distribution, its intrinsic geometry, and its geodesics-(Sec. II); and (2) to discuss in detail the dynamical behavior of Melvin's magnetic universe when it is subjected to arbitrarily large radial perturbations (Sec. III).

\section{PHYSICAL STRUCTURE OF MELVIN'S UNIVERSE \\ Magnetic Field and C-Energy Distributions}

The simplest metric, which fully exhibits the wholecylinder, static symmetry of Melvin's magnetic universe, is (Melvin I, II)

$$
\begin{array}{r}
d s^{2}=\bar{a}^{2}\left\{\left(1+\rho^{2}\right)^{2}\left(d \tau^{2}-d \rho^{2}-d \zeta^{2}\right)-\rho^{2}\left(1+\rho^{2}\right)^{-2} d \varphi^{2}\right\} \\
=\bar{a}^{2} d \sigma^{2}
\end{array}
$$

Here $\bar{a}$ is the "range-radius of the universe"; $\tau, \rho$, and $\zeta$ are dimensionless time, radial, and longitudinal coordinates; and $\varphi$ is the azimuthal angle. ${ }^{11}$ The magneticfield strength $\mathbf{B}$, and the $C$-energy density $\epsilon_{\mathrm{ce}}$, measured in a local Lorentz reference frame by an observer with world line $(\rho, \zeta, \varphi)=$ constant,$^{12}$ are

$$
\begin{aligned}
\mathbf{B}=\frac{2}{\bar{a} G^{1 / 2}} \frac{\mathbf{e}_{\zeta}}{\left(1+\rho^{2}\right)^{2}} & \\
= & \left(\frac{1}{1+\rho^{2}}\right)^{2}\left(\frac{1 \mathrm{~cm}}{\bar{a}}\right) \times 7 \times 10^{24} \mathrm{G}_{\zeta} \\
\epsilon_{\mathrm{ce}}=\frac{1}{2 \pi G \bar{a}^{2}} \frac{1}{1+\rho^{2}} & \\
= & \left(\frac{1}{1+\rho^{2}}\right)\left(\frac{1 \mathrm{~cm}}{\bar{a}}\right)^{2} \times 2 \times 10^{27} \mathrm{~g} / \mathrm{cm}^{3}
\end{aligned}
$$

They are plotted against proper radial distance in Fig. 1. On the axis of symmetry $\epsilon_{\mathrm{ce}}$ is the same as the magnetic energy density $\mathbf{B}^{2} / 8 \pi$, but far from the axis it is composed almost entirely of gravitational $C$ energy. The total $C$ energy per unit standard length ${ }^{13}$ contained

11 This coordinate system is the one of Melvin I, II. It differs from that of Thorne (Ref. 7) by the use of dimensionless variables : $\beta t \rightarrow \tau, \beta r \rightarrow \rho, \beta z \rightarrow \zeta, \beta=1 / \bar{a}$.

12 Such an observer needs the support of a rocket ship to prevent himself from falling down to the symmetry axis; alternatively, he could be momentarily stationary in free-fall at the apogee of a radial (vertical) orbit

${ }^{13}$ By "standard length" we mean that $\zeta$-coordinate interval $h_{\zeta}$, such that, when a system is in static equilibrium in the neighborhood of the symmetry axis, $\left[g_{\zeta \zeta}(\rho=0)\right]^{1 / 2} h_{\zeta}=$ one unit of proper length. For Melvin's magnetic universe-in the coordinates of Eq. (1) $-h_{\zeta}=1$, and (standard length) $=$ (coordinate length). (See Ref. 7.) 
in the cylinder $\rho<\rho_{o}$ of the hypersurface $\tau=$ constant $^{14}$ is

$$
\begin{aligned}
E\left(\rho_{o}\right)=\int_{0}^{\rho_{o}} \sqrt{ } g_{\rho \rho} d \rho \int_{0}^{2 \pi} \sqrt{ } g_{\varphi \varphi} & d \varphi \int_{0}^{h_{\zeta}} \sqrt{ } g_{\zeta \zeta} d \zeta \epsilon_{\mathrm{ce}} \\
& =(1 / 2 G) \ln \left(1+\rho_{o}^{2}\right) .
\end{aligned}
$$

It is this quantity which is minimized by Melvin's magnetic universe, as we shall see in Sec. III.

\section{Embedding Diagram and Geodesics}

The geometry of Melvin's magnetic universe is best exhibited by embedding ${ }^{15}$ the 2-dimensional surface $(\tau, \zeta)=$ constant in Euclidean 3-space. This surface has the form of a narrow-necked vase whose neck closes off asymptotically at an infinite distance from its base (Fig. 2).

At very large distances from the symmetry axis, Melvin's universe approaches Levi-Civita's ${ }^{16}$ solution for a line mass. According to Levi-Civita's definition, Melvin's universe has a total mass per unit length of $1 / G=1.35 \times 10^{28} \mathrm{~g} / \mathrm{cm}$; but according to the author's definition [Eq. (4)] it contains an infinite total $C$ energy per unit of the group-theoretically defined "standard length."17

The geodesics of Melvin's magnetic universe give additional insight into its physical structure. ${ }^{18}$ The geodesic equations for the line element (1) can be put

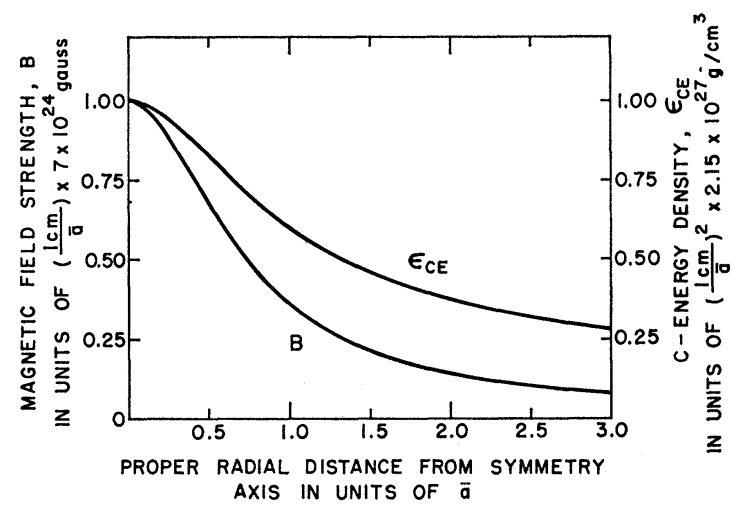

FIg. 1. The distribution of the magnetic-field strength $B$ and the $C$-energy density $\epsilon_{\mathrm{ce}}$ in Melvin's magnetic universe, as measured in local Lorentz frames by stationary observers (Ref. 11) [observers with world lines $(\rho, \zeta, \varphi)=$ constant].

${ }^{14}$ Just as the $C$-energy density seen by an observer with 4 velocity $v^{i}$ is $P^{i} v_{i}$, where $P^{i}$ is the $C$-energy-flux vector, so the total $C$ energy contained in a segment $S$ of a hypersurface is

(see Ref. 7).

$$
(C \text { energy in } S)=\int_{S} P^{i} d S_{i}=\int_{S} \epsilon_{\mathrm{ce}} d(\text { physical area })
$$

${ }^{15}$ For a discussion of embedding diagrams see, for instance, K. S. Thorne, Ref. 6

${ }^{16}$ T. Levi-Civita, Rend. Accad. Lincei 28, 3, 101 (1919).

${ }^{17}$ For a discussion of the relative merits of the two definitions, see Ref. 7.

${ }^{18} \mathrm{M}$. A. Melvin and J. S. Wallingford (to be published), have independently made a more complete study of the geodesics of Melvin's magnetic universe.

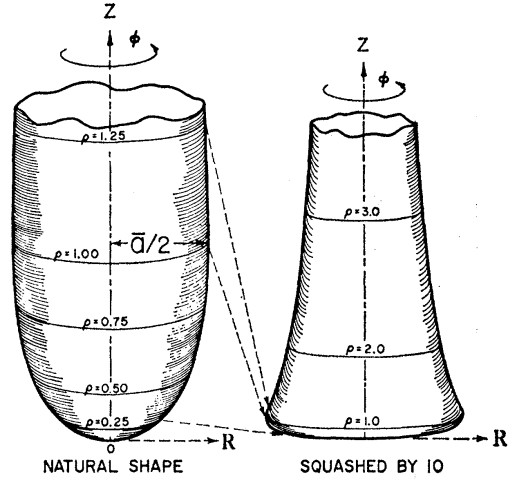

FIG. 2. Embedding diagram for Melvin's magnetic universe. The two-dimensional surface of this tall narrow-necked vase is the surface of constant $\tau$ and $\zeta$ embedded in Euclidean 3-space. For a different value of $\bar{a}$ the figure has the same shape but a different size. Thus, a large central magnetic field, $B_{\text {central }}=2 /\left(\bar{a} G^{1 / 2}\right)$, yields a figure of small maximum radius $R_{\max }=\bar{a} / 2$, while a small central field yields a large maximum radius. The neck of the "vase" closes off asymptotically as $\rho$ tends toward infinity. In order to exhibit this, it was necessary to squash the vase uniformly in the $Z$ direction in the second view.

in the form

$$
\begin{aligned}
& d \tau / d \sigma=\xi /\left(1+\rho^{2}\right)^{2}, \\
& d \zeta / d \sigma=\mu /\left(1+\rho^{2}\right)^{2}, \\
& d \varphi / d \sigma=\nu\left(1+\rho^{2}\right)^{2} / \rho^{2},
\end{aligned}
$$

$$
\begin{aligned}
\frac{d^{2} \rho}{d \sigma^{2}}+\frac{2 \rho}{1+\rho^{2}}\left(\frac{d \rho}{d \sigma}\right)^{2} & \\
& +2\left(\xi^{2}-\mu^{2}\right) \frac{\rho}{\left(1+\rho^{2}\right)^{5}}-\frac{\nu^{2}}{\rho^{3}}\left(\frac{1-\rho^{2}}{1+\rho^{2}}\right)=0,
\end{aligned}
$$

where $\xi, \mu, \nu$ are constants of integration and $d \sigma=d s / \bar{a}$. By solving these equations one finds that geodesics of constant $(\rho, \zeta)$ are given by

$$
\varphi= \pm\left[1+\rho^{2}\right]^{2}\left[2 /\left(1-\rho^{2}\right)\right]^{1 / 2} \tau .
$$

\begin{tabular}{|c|c|c|}
\hline$\rho$ & Geodesic character & $\begin{array}{l}\text { Proper circumference } \\
\text { of geodesic }\end{array}$ \\
\hline $0 \leq \rho<1 / \sqrt{3}$ & $\begin{array}{l}\text { time-like } \\
\text { (orbit of a particle) }\end{array}$ & $\begin{array}{c}\pi \sqrt{2} \bar{a}\left(1-3 \rho^{2}\right)^{1 / 2} /\left(1+\rho^{2}\right) \\
=\text { period of orbital } \\
\text { motion as measured } \\
\text { by particle }\end{array}$ \\
\hline$\rho=1 / \sqrt{3}$ & $\begin{array}{l}\text { light-like } \\
\text { (orbit of a photon) }\end{array}$ & 0 \\
\hline $\begin{array}{l}1 / \sqrt{3}<\rho \leq 1 \\
\rho>1\end{array}$ & $\begin{array}{l}\text { space-like } \\
\text { no circular geodesics exist }\end{array}$ & $\pi \sqrt{2} \bar{a}\left(3 \rho^{2}-1\right)^{1 / 2} /\left(1+\rho^{2}\right)$ \\
\hline
\end{tabular}

These geodesics, which are circles about the symmetry axis, have the character shown in Table I. In particular, for $\rho<1 / \sqrt{3},\left|g_{00} d \tau^{2}\right|>\left|g_{\varphi \varphi} d \varphi^{2}\right|$ on these geodesics, and they are time-like; but for $1 / \sqrt{3}<\rho<1, \quad\left|g_{00} d \tau^{2}\right|$

TABLE I. The character of circular geodesics $(\rho, \zeta$ constant) about the symmetry axis of Melvin' magnetic universe. These geodesics are described by Eq. (6). 


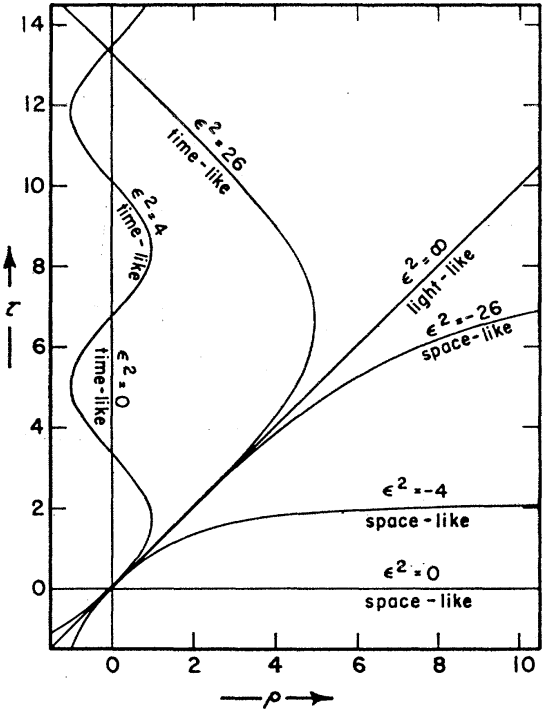

FIg. 3. Radial geodesics in Melvin's magnetic universe [Eq. (7)]. All time-like geodesics oscillate about the symmetry axis; all other geodesics extend to radial infinity. The vertical time-like geodesic, $\rho=0$, is mislabeled; it should be labeled $\epsilon^{2}=1$.

$<\left|g_{\varphi \varphi} d \varphi^{2}\right|$, and they are space like. Consequently, a particle can move in a circular orbit about the axis of symmetry only at radii $\rho<1 / \sqrt{3}$. At $\rho=1 / \sqrt{3}$, the gravitational deflection of light by the mass inside $\rho$ is sufficient to keep a photon in a circular orbit. For $\rho>1 / \sqrt{3}$ no particle or photon can move fast enough to stay in a circular orbit; the gravitational attraction toward the symmetry axis is too great.

The only geodesic of constant $(\rho, \varphi)$ is the null geodesic $\zeta= \pm \tau$. It is strange that, although a photon moving in the plane perpendicular to the axis of symmetry ( $\zeta=$ constant) is strongly deflected by the mass inside its orbit, a photon moving parallel to the axis of symmetry $(\rho, \varphi$ constant $)$ is not deflected at all..$^{19}$ The following consideration makes one feel more comfortable about this apparent paradox: Although Melvin's universe is invariant under translations along the $\zeta$ direction, $g_{\zeta \zeta}$ is not independent of $\rho$; to invariantly translate Melvin's universe, one must translate it by $\left(1+\rho^{2}\right)$ times as great a proper distance at $\rho$ as one does on the symmetry axis. Hence, an invariant translation of Melvin's universe is more like a rotation of Euclidean space than like a translation of it; and $\zeta$ is more like an angular coordinate of Euclidean space than like a rectilinear coordinate. Just as it requires mass to deflect a photon into circular motion in Euclidean space, so it takes mass to "deflect" one into motion along a $\zeta$-coordinate line in Melvin's universe. "Undeflected motion" would correspond to some path other than $\zeta=$ constant.

19 The same thing happens to photons moving in the gravitational field of a pencil of light - see R. C. Tolman, Relativity, Thermodynamics, and Cosmology (Clarendon Press, Oxford, 1934), p. 274.
Radial geodesics $(\zeta, \varphi$ constant $)$ are described by

$$
\begin{gathered}
d \rho / d \tau= \pm\left[1-\left(1+\rho^{2}\right)^{2} / \epsilon^{2}\right]^{1 / 2}, \\
\epsilon^{2} \text { a constant } \geq 1 \text {, or } \leq 0 .
\end{gathered}
$$

$\epsilon^{2} \geq 1$ corresponds to time-like, $\epsilon^{2}=0$ to light-like, and $\epsilon^{2}<0$ to space-like geodesics. (See Fig. 3.) It is evident that the gravitational attraction toward the center of the universe is great enough to prevent any object of nonzero rest mass from escaping to radial infinity. ("Escape velocity" is infinite.) However, any radially moving photon "eventually" reaches radial infinity.

This completes our discussion of the physical structure of Melvin's magnetic universe.

\section{DYNAMICAL BEHAVIOR OF MELVIN'S MAGNETIC UNIVERSE WHEN PERTURBED}

\section{Notation and Background Equations}

In discussing the dynamical behavior of Melvin's magnetic universe when perturbed radially, we use the "hyperbolic canonical coordinate system"

$$
\begin{aligned}
d s^{2}=\bar{a}^{2}\left\{e^{2(\gamma-\psi)}\left(d \tau^{2}-d \rho^{2}\right)-e^{2 \psi} d \zeta^{2}-\rho^{2} e^{-2 \psi} d \varphi^{2}\right\} & =\bar{a}^{2} d \sigma^{2} .
\end{aligned}
$$

In this coordinate system, ${ }^{21}$ the gauge can be chosen so that the only nonvanishing components of the electromagnetic vector potential are $A_{2}$ and $A_{3}$, and the only nonvanishing components of the electromagnetic field tensor are

$$
\begin{array}{ll}
f_{02}=-f_{20}=\bar{a} G^{-1 / 2} \dot{A_{2}}, & f_{12}=-f_{21}=\bar{a} G^{-1 / 2} A_{2^{\prime}}, \\
f_{03}=-f_{30}=\bar{a} G^{-1 / 2} \dot{A_{3}}, & f_{13}=-f_{31}=\bar{a} G^{-1 / 2} A_{3^{\prime}} .
\end{array}
$$

Here $\dot{A}_{j}=\partial A_{j} / \partial \tau, A_{j}{ }^{\prime}=\partial A_{j} / \partial \rho$. The electric and magnetic fields measured in a local Lorentz frame by an observer with world line $(\rho, \zeta, \varphi)=$ constant are ${ }^{11}$

$$
\begin{aligned}
& \mathbf{B}=-\left(\bar{a} G^{1 / 2}\right)^{-1}\left[\left(A_{3}{ }^{\prime} / \rho\right) e^{2 \psi-\gamma} \mathbf{e}_{\zeta}-A_{2}{ }^{\prime} e^{-\gamma} \mathbf{e}_{\varphi}\right], \\
& \mathbf{E}=-\left(\bar{a} G^{1 / 2}\right)^{-1}\left[\left(\dot{A}_{3} / \rho\right) e^{2 \psi-\gamma} \mathbf{e}_{\varphi}+\dot{A}_{2} e^{-\gamma} \mathbf{e}_{\zeta}\right],
\end{aligned}
$$

and the total magnetic flux inside $\rho$ is

$$
\begin{aligned}
\Phi(\rho)=\int_{0}^{\rho} \int_{0}^{2 \pi} B_{\zeta}\left(g_{\rho \rho} g_{\varphi \varphi}\right)^{1 / 2} d \varphi d \rho \\
\quad=(2 \pi \bar{a}) G^{-1 / 2}\left[A_{3}(0)-A_{3}(\rho)\right] .
\end{aligned}
$$

In the canonical coordinate system (8), the EinsteinMaxwell field equations reduce to

$$
\begin{gathered}
\ddot{\psi}-(1 / \rho)\left(\rho \psi^{\prime}\right)^{\prime}=-e^{-2 \psi}\left(\dot{A_{2}}{ }^{2}-A_{2}{ }^{2}\right) \\
+\left(e^{2 \psi} / \rho^{2}\right)\left({\dot{A_{3}}}^{2}-A_{3}{ }^{2}\right), \\
\ddot{A}_{2}-(1 / \rho)\left(\rho A_{2}{ }^{\prime}\right)^{\prime}=2\left(\dot{\psi} \dot{A}_{2}-\psi^{\prime} A_{2}{ }^{\prime}\right), \\
\ddot{A}_{3}-\rho\left(A_{3}{ }^{\prime} / \rho\right)^{\prime}=-2\left(\dot{\psi} \dot{A}_{3}-\psi^{\prime} A_{3}{ }^{\prime}\right),
\end{gathered}
$$

${ }^{20}$ Melvin II (Ref. 2), and Thorne (Ref. 7). This coordinate system differs from that of Ref. 7 in that the constant $\bar{a}$ has been factored out of the line element.

${ }^{21}$ For proof of the statements in this paragraph, see Melvin II, and Thorne (Ref. 7). 


$$
\begin{gathered}
\gamma^{\prime}=\rho\left(\dot{\psi}^{2}+\psi^{\prime 2}\right)+\rho e^{-2 \psi}\left({\dot{A_{2}}}^{2}+{A_{2}}^{\prime 2}\right) \\
+\left(e^{2 \psi} / \rho\right)\left({\dot{A_{3}}}^{2}+{A_{3}}^{\prime 2}\right) \\
\dot{\gamma}=2 \rho \dot{\psi} \psi^{\prime}+2 \rho e^{-2 \psi} \dot{A}_{2}{A_{2}}^{\prime} \\
+2\left(e^{2 \psi} / \rho\right){\dot{A_{3}}}_{A_{3}}{ }^{\prime} .
\end{gathered}
$$

Note that the structure of any cylindrical electromagnetic universe is completely determined by the gravitational and electromagnetic potential functions, $\psi$ and $A_{j}$. Once they are known, everything else is determined via Eqs. (12d), (12e), (8), (9), (10), and (11). Melvin's magnetic universe corresponds to

$$
\begin{aligned}
\psi & =\ln \left(1+\rho^{2}\right), \\
A_{2} & =0, \\
A_{3} & =1 /\left(1+\rho^{2}\right), \\
\gamma & =2 \ln \left(1+\rho^{2}\right) .
\end{aligned}
$$

The $C$-energy density measured in a local Lorentz frame by an observer with world line $(\rho, \zeta, \varphi)=$ constant $^{12}$ is

$$
\epsilon_{\mathrm{ce}}=\left(8 \pi G \bar{a}^{2} h_{\zeta}\right)^{-1}\left(e^{\psi-\gamma} / \rho\right) \gamma^{\prime}
$$

and the total $C$ energy per unit standard length ${ }^{13}$ contained inside the cylinder of coordinate radius $\rho$ and on the hypersurface $\tau=$ constant is

$$
E(\rho)=(1 / 4 G) \gamma(\rho)=K(\rho)+P(\rho),
$$

where $K$, the kinetic $C$ energy, and $P$, the potential $C$ energy per unit standard length, are

$$
\begin{aligned}
& K(\rho)=\left(\frac{1}{4 G}\right) \int_{0}^{\rho}\left[\rho \dot{\psi}^{2}+\rho e^{-2 \psi}{\dot{A_{2}}}^{2}+\left(e^{2 \psi} / \rho\right) \dot{A}_{3}{ }^{2}\right] d \rho, \\
& P(\rho)=\left(\frac{1}{4 G}\right) \int_{0}^{\rho}\left[\rho \psi^{\prime 2}+\rho e^{-2 \psi}{A_{2}}^{2}+\left(e^{2 \psi} / \rho\right) A_{3}{ }^{2}\right] d \rho,
\end{aligned}
$$

[cf. Eqs. (12) and (15)].

Melvin's magnetic universe is that configuration of gravitational and electromagnetic potential fields, $\psi(\rho, t)$, $A_{j}(\rho, t)$, which absolutely minimizes the $C$ energies $E\left(\rho_{o}\right)$ and $P\left(\rho_{o}\right)$ inside any radius $\rho_{o}$ on the hypersurface $t=$ constant, subject to the constraint of fixed magnetic flux $\Phi\left(\rho_{o}\right)$, and fixed gravitational potential, $\psi\left(\rho_{o}\right)$, at $\rho_{o}$. This $C$-energy minimum property is proved in Ref. 7, and forms the foundation for the discussion of the dynamical behavior of Melvin's magnetic universe, when it is perturbed.

\section{The Form of the Perturbation}

Let Melvin's universe be perturbed radially in an arbitrary (but physically reasonable) manner on the hypersurface $\tau=0$. But confine the perturbation to a finite region $\rho<R$ about the symmetry axis, so that the potential functions $\psi$ and $A_{j}$ are identical to Melvin's potential functions outside $\rho=R$ :
Initial conditions at ${ }^{22} \tau=0$

(1) For $\rho \leq R$ :

$\psi(\rho, 0), \psi(\rho, 0), A_{j}(\rho, 0), \dot{A_{j}}(\rho, 0)$ arbitrary except for the conditions

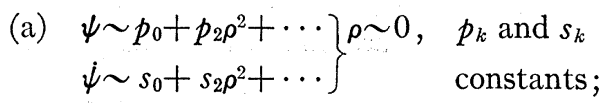

(b) $A_{2} \sim a_{20}+a_{22} \rho^{2}$

$A_{3} \sim a_{30}+a_{32} \rho^{2} \cdots \quad \rho \sim 0, \quad a_{j k}$ and $b_{j k}$

$\left.\dot{A_{2}} \sim b_{20}+b_{22} \rho^{2} \cdots\right\} \quad$ constants;

$\dot{A}_{3} \sim b_{22} \rho^{2} \ldots$

(c) $\psi^{\prime}(\rho, 0)$ continuous ;

(d) $A_{j}(\rho, 0)$ piece-wise smooth.

(2) For $\rho \geq R$ :

$$
\begin{aligned}
\psi & =\ln \left(1+\rho^{2}\right), \\
A_{2} & =0, \\
A_{3} & =1 /\left(1+\rho^{2}\right),
\end{aligned}
$$

(3) For $\rho=R$ :

$$
\psi, \psi^{\prime} \text {, and } A_{j} \text { continuous }
$$

(4) For all $\rho$ :

$$
\begin{aligned}
\gamma=\int_{0}^{\rho}\left[\rho\left(\dot{\psi}^{2}+\psi^{\prime 2}\right)+\rho e^{-2 \psi}\left({A_{2}}^{2}+A_{2}{ }^{2}\right)\right. & \\
& \left.+\left(\frac{e^{2 \psi}}{\rho}\right)\left({\dot{A_{3}}}^{2}+{A_{3}}^{\prime 2}\right)\right] d \rho
\end{aligned}
$$

\section{Constants of the Motion}

Let such a perturbed universe be followed over the period of time $0 \leq \tau \leq \tau_{o}$. One finds that, for any $\bar{R}>R+\tau_{o}, A_{2}(\bar{R}), A_{3}(\bar{R}), \psi(\bar{R}), \gamma(\bar{R}), A_{3}(0)$, and $\gamma(0)=0$ are all constants of the motion; and consequently, the total magnetic flux and the total $C$ energy per unit standard length inside $\bar{R}$ are both conserved. $\left[A_{j}(\bar{R})\right.$, $\psi(\bar{R})$, and $\gamma(\bar{R})$ are constant because information of the existence of the perturbation cannot travel faster than the speed of light; $A_{3}(0)$ and $\gamma(0)$ are constant by virtue of the field equations (12) and the initial conditions (17). Conservation of flux and $C$ energy follow from their definitions, Eqs. (11) and (15).]

${ }^{22} \psi, \dot{\psi}, A_{2}, A_{2}, A_{3}, \dot{A_{3}}$ must be even in $\rho$ because the C. E. U. is invariant under reflections through its axis of symmetry and because the longitudinal electric and magnetic fields on the axis must be finite and the axial fields zero. One might think the absence of terms linear in $\rho$ would be enough; however, the field equations, (12a)-(12c), link the coefficients of odd powers of $\rho$ together in such a way that, unless they all vanish initially, terms linear in $\rho$ will soon become nonzero. $A_{3}(\rho=0)=0$ in order to keep the electric field finite on the axis. Continuity of $\psi$ and $\psi^{\prime}$ arises from Lichnerowicz's junction conditions [A. Lichnerowicz, Théories relativistes de la gravitation et de l'électromagnétisme (Masson et Cie, Paris, 1955) ]. $A_{j}$ is piece-wise smooth because it is a potential function. 
One finds that the total kinetic $C$ energy per unit standard length in the entire universe, $K(\infty)$, has an upper bound:

$$
\begin{aligned}
& K(\infty) \leq E(\rho=R, \tau=0)- \ln \left(1+R^{2}\right) / 2 G \\
&=(C \text { energy per unit standard length } \\
&\text { associated with the perturbation }) .
\end{aligned}
$$

This follows directly from the $C$ energy minimum property of Melvin's universe.

\section{Collapse and Explosion Forbidden}

Suppose that the initial perturbation were strong enough and in the right direction to initiate gravitational collapse of part or all of the active region of the universe. Let $\Phi_{o}$ be the magnetic flux carried into oblivion during the collapse, and call the cylinder containing a flux of exactly $\Phi_{o}$ the "collapsing region" of the universe. One can show (Appendix A) that, when collapse has gone so far that the proper radius $s$ of the collapsing region is much less than $\left(G^{1 / 2} / 2 \pi\right)\left|\Phi_{o}\right|$, then the difference between the total $C$ energies in the collapsing universe and in the unperturbed universe is

$$
\begin{aligned}
& \lim _{\rho \rightarrow \infty}\left[E_{\text {collapse }}(\rho)-E_{\text {unpert }}(\rho)\right] \\
& =(C \text { energy per unit standard length } \\
& \text { associated with the perturbation }) \\
& \geq(8 \pi \sqrt{ } G)^{-1}\left(\left|\Phi_{o}\right| / s\right) .
\end{aligned}
$$

Consequently, in order to initiate collapse $(s \rightarrow 0)$, one must supply an infinite $C$ energy through the perturbation. But this cannot be done with a perturbation which is confined to a finite region about the axis of symmetry. ${ }^{23}$ Consequently, gravitational collapse cannot be initiated by a radial perturbation of Melvin's universe.

Nor can electromagnetic explosion be initiated by a radial perturbation. For, consider a stage of explosion in which essentially all the electromagnetic and gravitational fields are evacuated from a region of proper radius $s \gg \bar{a}$ about the symmetry axis. When this stage of explosion has been reached, the excess $C$ energy in the exploding universe over that in the unperturbed universe is

$$
\begin{array}{r}
\lim _{\rho \rightarrow \infty}\left[E_{\text {explode }}(\rho)-E_{\text {unpert }}(\rho)\right] \\
=(C \text { energy per unit standard length } \\
\text { associated with the perturbation }) \\
\geq(1 / G) \ln (s / \bar{a}) .
\end{array}
$$

\footnotetext{
${ }^{23}$ Cylindrical systems which contain an infinite amount of $C$ energy per unit standard length inside a finite region about the axis of symmetry are discussed very briefly in Ref. (7) and in great detail in (1) K. S. Thorne, Ph.D. thesis, Princeton University, 1965 (unpublished); and (2) K. S. Thorne, Geometrodynamics of Cylindrical Systems (book in preparation). According to analyses presented in these two references, no perturbation of Melvin's universe, which is confined to a finite region about the axis of symmetry, can have infinite $C$-energy associated with it. Any perturbation with infinite $C$-energy profoundly alters Melvin's universe all the way out to radial infinity.
}

(See Appendix A for proof.) Consequently, a stage of complete dispersion of the electromagnetic and gravitational fields of Melvin's universe $(s \rightarrow \infty)$ can never be reached as the result of a finite radial perturbation, for the state of completely dispersed magnetic field lines has infinitely more $C$ energy per unit standard length than does Melvin's universe.

\section{Actual Dynamical Behavior}

If no radial perturbations of Melvin's magnetic universe can initiate gravitational collapse or electromagnetic explosion of the magnetic field lines, just what are the effects of radial perturbations? For insight into this question, we turn again to the $C$-energy properties of perturbed cylindrical electromagnetic universes.

One can show ${ }^{24}$ that the $C$ energy per unit standard length $E\left[\psi, A_{j}\right]$, behaves, in all respects, as the energy for two coupled fields normally behaves: Its mathematical form [Eqs. (16)] is that of a field energy; it can be split into kinetic part $K$ plus potential part $P ; K-P$ is a Lagrangian for the Eqs. (12a)-(12c) governing the dynamics of the coupled fields; these dynamical equations are coupled wave equations; and $P+K=E$ is minimized by static configurations. This perfectly normal behavior of $C$ energy under all tests we have made of it suggests the following conjecture: If the fields $\psi$ and $A_{j}$ are strongly distorted inside a radius $\rho=R$ and then released, they will do the normal thing: The fields inside $\rho=R$ will undergo damped, turbulent oscillation, emitting the $C$ energy associated with the perturbation as gravitational and electromagnetic radiation. As the $C$ energy is gradually lost from the active region, the motion of the fields there will become less and less violent, until finally they will settle down into Melvin's static, unperturbed configuration. All that will be left of the perturbation will be a series of cylindrical gravitational and electromagnetic waves traveling off toward infinity. These waves, which carry away the excess $C$ energy, have 3 degrees of freedom -2 corresponding to the 2 polarization directions of electromagnetic waves, and 1 corresponding to the single polarization direction allowed by whole-cylinder symmetry for gravitational waves.

That this is, indeed, the dynamical behavior of Melvin's universe when perturbed radially is supported not only by analogy with other cases of coupled, classical fields, but also by the fact that, if the gravitational field around a static cylinder $\left(A_{j}=0\right)$ is perturbed, a similar behavior is observed. ${ }^{25}$ The unperturbed static background field, $\psi_{o}=\kappa \ln \rho+b$, and the perturbation, $\psi_{1}(\rho, \tau)$, are totally oblivious of each other. $\psi_{1}(\rho, \tau)$ propagates according to the homogeneous wave equation; and being initially confined within $\rho=R$, it eventually propagates off toward $\rho=\infty$. Additional support for our conjecture comes from the analysis of

${ }^{24}$ See Ref. 7, and Sec. I of this paper.

${ }^{25}$ See Sec. IV. C. of Ref. 7. 
small perturbations of Melvin's magnetic universe by Melvin II. His results indicate that small perturbations confined initially to a finite region $\rho<R$ eventually propagate off to $\rho=\infty$, superposed linearly on the static solution.

Finally, the validity of our conjecture has been verified for several arbitrarily chosen, very large perturbations, by numerical solution of the EinsteinMaxwell equations (12). ${ }^{26}$ An example is presented in Fig. 4.

\section{Relation of C-Energy Analysis to Small Perturbation Analysis}

Our discussion of the stability of Melvin's magnetic universe against radial perturbations would be incomplete without a mention of the relation of the $C$ energy proof of stability presented here to the small perturbations proof of Melvin II. Melvin solves the linearized equations for small departures from the static configuration to obtain the normal modes of the system. He finds that the physically acceptable normal modes are all oscillatory rather than exponentially growing, and from this he concludes that his model universe is stable against small radial perturbations.

The small perturbation problem can be approached alternatively from the $C$-energy point of view. Let the departures of the gravitational and electromagnetic potentials from their equilibrium values be $\psi^{*}(\rho, \tau)$ and $A_{\jmath}{ }^{*}(\rho, \tau)$ :

$$
\begin{aligned}
\psi(\rho, \tau) & =\ln \left(1+\rho^{2}\right)+\psi^{*}(\rho, \tau), \\
A_{2}(\rho, \tau) & =0+A_{2}{ }^{*}(\rho, \tau), \\
A_{3}(\rho, \tau) & =1 /\left(1+\rho^{2}\right)+A_{3}{ }^{*}(\rho, \tau) .
\end{aligned}
$$

Expand the potential and kinetic $C$ energies per unit standard length on the canonical hypersurfaces to second order in $A_{j}^{*}(\rho, \tau)$ :

$$
\begin{aligned}
P\left(\rho_{o}, \tau\right)- & (1 / 2 G) \ln \left(1+\rho_{0}^{2}\right)=\rho^{*}\left[\psi^{*}, A_{j}^{*}\right] \\
= & \frac{1}{4 G} \int_{0}^{\rho_{o}}\left\{\frac{8 \rho}{\left(1+\rho^{2}\right)^{2}} \psi^{*_{2}}+\rho \psi^{* / 2}+\frac{\rho}{\left(1+\rho^{2}\right)^{2}} A_{2}{ }^{* / 2}\right. \\
& \left.+\frac{\left(1+\rho^{2}\right)^{2}}{\rho} A_{3}^{* / 2}-8 \psi^{*} A_{3}{ }^{* \prime}\right\} d \rho \\
K\left(\rho_{o}, \tau\right)= & \mathcal{K}^{*}\left[\dot{\psi}^{*}, \dot{A}_{j}^{*}\right] \quad \dot{A}_{2}^{{ }^{*}} \\
= & \frac{1}{4 G} \int_{0}^{\rho_{o}}\left\{\rho \dot{\psi}^{* 2}+\frac{\rho}{\left(1+\rho^{2}\right)^{2}}+\frac{\left(1+\rho^{2}\right)^{2}}{\rho} \dot{A_{3}{ }^{* 2}}\right\} d \rho .
\end{aligned}
$$

${ }^{26}$ The finite-difference approximation used in these calculations is briefly described in Appendix $\mathbf{B}$.

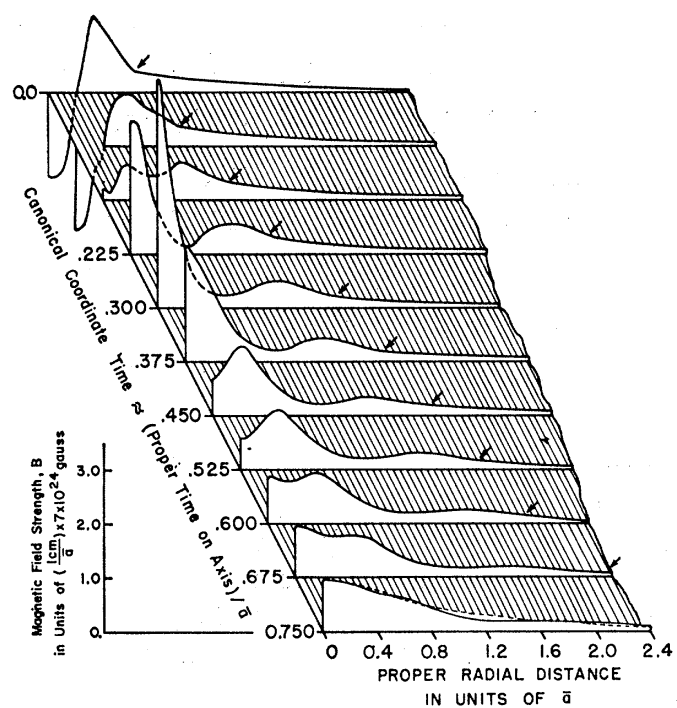

FIG. 4. Longitudinal magnetic-field strength $B_{\zeta}$ as a function of proper radial distance and canonical coordinate time, for a particular strongly perturbed version of Melvin's magnetic universe. The crosshatched plane is $B_{\zeta}=0$. The arrows indicate the wave front dividing the perturbed region of the universe (left) from the unperturbed region (right). At (coordinate time) $=0.750$ two configurations are shown: The solid line is the actual configuration at that time, while the dashed line is Melvin's unperturbed configuration, toward which the universe is evolving. The initial perturbation is time-symmetric $\left(\dot{\psi}=\dot{A_{j}}=\dot{\gamma}=0\right)$; it has $E_{\zeta}=B_{\varphi}=0\left(A_{2}=\dot{A}_{2}=0\right)$ everywhere; and it is confined to the region $\rho<R=0.5$ (proper radial distance $<0.542 \bar{a}$ ). The total $C$ energy per unit standard length inside $R$ at $t=0$ is $E(R)=3.1 \times 10^{27}$ $\mathrm{g} / \mathrm{cm}$, as compared to a value of $1.5 \times 10^{27} \mathrm{~g} / \mathrm{cm}$ for the unperturbed configuration. Consequently, the total $C$ energy which must be radiated is $1.6 \times 10^{27} \mathrm{~g} / \mathrm{cm}$. In the figure, one can see how the magnetic field oscillates turbulently, throwing off its excess $C$ energy in the form of electromagnetic and gravitational waves, as it gradually settles down into Melvin's static configuration.

Since $K-P$ is a Lagrangian for the dynamical equations governing $\psi$ and $A_{j}, \mathfrak{K}^{*}-\odot^{*}$ is a Lagrangian for those governing $\psi^{*}$ and $A_{j}^{*}$

$$
\begin{aligned}
\delta \rho^{*} / \delta \psi^{*} & =-(1 / 4 G) 2 \rho \ddot{\psi}^{*}, \\
\delta \rho^{*} / \delta A_{2}{ }^{*} & =-(1 / 4 G) 2\left[\rho /\left(1+\rho^{2}\right)^{2}\right] \ddot{A}_{2}{ }^{*}, \\
\delta \rho^{*} / \delta A_{3}{ }^{*} & =-(1 / 4 G) 2\left[\left(1+\rho^{2}\right) / \rho\right] \ddot{A}_{3}{ }^{*} .
\end{aligned}
$$

The coupled eigenvalue equations satisfied by the space parts of the normal modes of the perturbed universe are obtained from Eqs. (21) by setting $\psi^{*}(\rho, \tau)=e^{i \omega \tau} \psi_{s}^{*}(\rho)$, $A_{j}^{*}(\rho, \tau)=e^{i \omega \tau} A_{j s}^{*}(\rho)$,

$$
\begin{aligned}
\delta \odot^{*} / \delta \psi_{s}{ }^{*} & =\omega^{2}(1 / 4 G) 2 \rho \psi_{s}{ }^{*}, \\
\delta \odot^{*} / \delta A_{2 s}{ }^{*} & =\omega^{2}(1 / 4 G) 2\left[\rho /\left(1+\rho^{2}\right)^{2}\right] A_{2 s}{ }^{*}, \\
\delta \odot^{*} / \delta A_{3 s}{ }^{*} & =\omega^{2}(1 / 4 G) 2\left[\left(1+\rho^{2}\right)^{2} / \rho\right] A_{3 s}{ }^{*} .
\end{aligned}
$$

These eigenvalue equations are the Euler-Lagrange equations corresponding to the variational principle

$$
\delta \odot^{*}\left[\psi_{s}^{*}, A_{j s}{ }^{*}\right]-\omega^{2} \delta \mathcal{K}^{*}\left[\psi_{s}^{*}, A_{j s}{ }^{*}\right]=0 .
$$


Consequently, the nth normal mode of radial motion about Melvin's equilibrium confuguration is given by

$$
\begin{aligned}
\psi(\rho, \tau) & =\ln \left(1+\rho^{2}\right)+\psi_{(n)}{ }^{*}(\rho) e^{i \omega_{n} \tau}, \\
A_{2}(\rho, \tau) & =0+A_{2(n)}{ }^{*}(\rho) e^{i \omega_{n} \tau}, \\
A_{3}(\rho, \tau) & =1 /\left(1+\rho^{2}\right)+A_{3(n)}{ }^{*}(\rho) e^{i \omega_{n} \tau},
\end{aligned}
$$

where $\psi_{(n)}{ }^{*}(\rho)$ and $A_{j(n)}{ }^{*}(\rho)$ are those functions which give the nth smallest extremal value of

$$
\omega^{2}=\lim _{\rho_{0} \rightarrow \infty} \Theta^{*}\left[\psi^{*}, A_{3}{ }^{*}\right] / \mathcal{K}^{*}\left[\psi^{*}, A_{3}{ }^{*}\right],
$$

and where $\omega_{n}$ is the square root of this nth smallest value. Of course we must require that $\psi^{*}$ and $A_{j}{ }^{*}$ be physically acceptable perturbations. ${ }^{27}$ In particular, $\psi^{*}(0)$ must be finite (geometry of space-time regular on symmetry axis); $A_{2}{ }^{*}(0)$ must be finite and $A_{3}{ }^{*}(0)$ must vanish (electric field finite on symmetry axis); $\psi^{*}(\infty)$ must vanish (perturbation in geometry of spacetime must die out at infinity); $A_{3}{ }^{*}(\infty)$ must be finite (total magnetic flux in universe finite); $A_{2}{ }^{* \prime} / \rho^{3} \rightarrow 0$ and $\rho^{3} A_{3}{ }^{* \prime} \rightarrow 0$ at infinity (perturbed electric field must die out at infinity). These boundary conditions, combined with the variational principle (25), determine the normal modes for small radial perturbations of Melvin's universe.

The stability of Melvin's magnetic universe against small radial perturbations is equivalent to the requirement that $\omega_{0}^{2}$, the lowest eigenfrequency squared, be positive. But it is positive, since the denominator of Eq. (25) is positive definite, and the minimum $C$-energy property of Melvin's magnetic universe demands that the numerator be positive for all choices of $\psi^{*}$ and $A_{j}{ }^{*}$.

Hence, we see that the nonexistence of exponentially growing normal modes is equivalent to the fact that Melvin's universe gives a local minimum of the $C$ energy per unit standard length subject to the physical constraints imposed on the normal modes at the symmetry axis and at infinity. Since Melvin's universe actually gives an absolute minimum of the $C$ energy, it is stable against large perturbations as well as against small ones.

This $C$-energy analysis of the stability of Melvin's magnetic universe against small radial perturbations is completely analogous to the author's recent energybased stability analysis for superdense stars. ${ }^{28}$

\section{ACKNOWLEDGMENTS}

The author is indebted to Professor J. A. Wheeler for suggesting this problem, and for many helpful discussions during its execution. He also wishes to thank Professor M. A. Melvin for a helpful exchange of ideas. The numerical computations were performed on the Princeton University IBM-7094 computer, which is

${ }^{27}$ For a detailed discussion of the restrictions on $\psi^{*}$ and $A_{j}{ }^{*}$, see Melvin II.

${ }^{28}$ See appendix B of B. K. Harrison, K. S. Thorne, M. Wakano, and J. A. Wheeler, Gravitation Theory and Gravitational Collapse (University of Chicago Press, Chicago, 1965). supported in part by National Science Foundation Grant NSF-GP 579.

\section{APPENDIX A : INFINITE $C$-ENERGY INPUT REQUIRED TO INDUCE COLLAPSE OR EXPLOSION \\ Collapse}

We here show that, if Melvin's universe is perturbed radially within a finite region about the symmetry axis, and if, as a result of the perturbation, at some later canonical coordinate time $\tau_{o}$, a longitudinal magnetic flux $\Phi_{o}$ has become concentrated within a proper radius $s \ll G^{1 / 2} \Phi_{o}$, about the symmetry axis, then the $C$ energy per unit standard length associated with the perturbation must have been

$$
\begin{aligned}
E_{\mathrm{pert}}=\lim _{\rho_{o} \rightarrow \infty}\left[E\left(\rho_{o}\right)-(1 / G) \ln \rho_{o}\right]_{\tau=\tau_{o}} \\
\\
\quad \geq(4 \pi \sqrt{ } G)^{-1}\left(\left|\Phi_{o}\right| / s\right) .
\end{aligned}
$$

To see this, let $\rho_{o} \gg 1$ be fixed at some point which, at time $\tau_{o}$, has not yet received word of the perturbation; and let $\rho_{s}$ be the radial coordinate corresponding to proper radial distance $s$. Then at time $\tau_{o} \psi$ and $A_{3}$ must satisfy the boundary conditions

$$
\begin{aligned}
\psi\left(\rho_{o}\right) & =\ln \left(1+\rho_{o}^{2}\right)=2 \ln \rho_{o}, \\
A_{3}(0)-A_{3}\left(\rho_{s}\right) & =\left(G^{1 / 2} / 2 \pi \bar{a}\right) \Phi_{o} .
\end{aligned}
$$

According to Eqs. (15) and (16), at time $\tau_{o}$

$$
E\left(\rho_{o}\right) \geq(1 / 4 G) \int_{0}^{\rho_{o}}\left\{\rho \psi^{\prime 2}+\left(e^{2 \psi} / \rho\right) A_{3}^{2}\right\} d \rho .
$$

Introducing new variables $x, y, l$ defined by

$$
x=A_{3}, \quad y=\rho e^{-\psi}, \quad d l=e^{-\psi} d \rho, \quad l(\rho=0)=0
$$

converts this to

$$
E\left(\rho_{o}\right) \geq(1 / 4 G) \int_{0}^{l_{o}}(1 / y)\left[(1-d y / d l)^{2}+(d x / d l)^{2}\right] d l .
$$

Let $y_{m}$ be the maximum value of $y(l)$ over the interval $\left(0, l_{s}\right)$, and let it occur at $l=l_{m}$. Then

$$
\begin{aligned}
E\left(\rho_{o}\right) \geq \frac{1}{4 G}\left\{\frac{1}{y_{m}} \int_{0}^{l_{m}}\left(1-\frac{d y}{d l}\right)^{2} d l\right. & \\
& \left.+\frac{1}{y_{m}} \int_{0}^{l_{s}}\left(\frac{d x}{d l}\right)^{2} d l+\int_{l_{m}}^{l_{o}} 1\left(1-\frac{d y}{d l}\right)^{2} d l\right\} .
\end{aligned}
$$

Applications of Schwarz's inequality transform this to

$$
E\left(\rho_{o}\right) \geq \frac{1}{4 G}\left\{\frac{\left(l_{m}-y_{m}\right)^{2}}{l_{m} y_{m}}+\frac{G}{4 \pi^{2} \bar{a}^{2}} \frac{\Phi_{o}{ }^{2}}{l_{s} y_{m}}+\int_{\rho_{m}}^{\rho_{o}} \rho \psi^{\prime 2} d \rho\right\} .
$$

The last integral is minimized when $\psi=\kappa \ln \left(\rho / \rho_{m}\right)$ $+\psi\left(\rho_{m}\right)$, where $\kappa=\left[\ln \left(\rho_{o}^{2} y_{m} / \rho_{m}\right)\right] /\left[\ln \left(\rho_{o} / \rho_{m}\right)\right]$. (Cf. 
Ref. 7, Sec. IV-B.) Consequently, whatever the quantities $\rho_{m}, \rho_{s}, \rho_{o}$, and $y_{m}$ may be,

$$
\begin{aligned}
E\left(\rho_{o}\right) \geq \frac{1}{4 G}\left\{-2+\frac{y_{m}}{l_{m}}+\frac{G}{4 \pi^{2} \bar{a}^{2}}\right. & \frac{\Phi_{o}^{2}}{l_{s} y_{m}} \\
& \left.+\frac{\left[\ln \left(\rho_{0}^{2} y_{m} / \rho_{m}\right)\right]^{2}}{\ln \left(\rho_{o} / \rho_{m}\right)}\right\} .
\end{aligned}
$$

However, since $s / \bar{a} \geq l_{s} \geq l_{m}$, Eq. (A5) implies

$$
\begin{aligned}
E\left(\rho_{o}\right) \geq \frac{1}{4 G}\left\{-2+\frac{y_{m}}{s / \bar{a}}+\frac{G}{4 \pi^{2} \bar{a}^{2}}\right. & \frac{\Phi_{o}{ }^{2}}{y_{m}(s / \bar{a})} \\
& \left.+\frac{\left[\ln \left(\rho_{o}^{2} y_{m} / \rho_{m}\right)\right]^{2}}{\ln \left(\rho_{o} / \rho_{m}\right)}\right\} .
\end{aligned}
$$

Now, if $\rho_{o}$ is not greater than $1 / y_{m}$, select a new value of $\rho_{o}$ which is. Then minimize expression (A6) with respect to $\rho_{m}$ and $y_{m}$ to obtain

$$
\begin{gathered}
E\left(\rho_{o}\right) \geq \frac{1}{4 G}\left\{-2+\frac{u}{s / \bar{a}}+\frac{G}{4 \pi^{2} \bar{a}^{2}} \frac{\Phi_{o}^{2}}{u(s / \bar{a})}+4 \ln \left(\rho_{o} u\right)\right\}, \\
u=\left[\left(G / 4 \pi^{2} \bar{a}^{2}\right) \Phi_{o}^{2}+4(s / \bar{a})^{2}\right]^{1 / 2}-2 s / \bar{a} .
\end{gathered}
$$

For $s \ll G^{1 / 2} \Phi_{o}$, this becomes

$$
E\left(\rho_{o}\right)-(1 / G) \ln \rho_{o} \geq(8 \pi \sqrt{ } G)^{-1}\left|\Phi_{o}\right| / s,
$$

which then yields Eq. (A1),

\section{Explosion}

Q.E.D.

Next, consider a perturbation of Melvin's universe which results in an explosion of the magnetic field lines away from the symmetry axis to the point that, at coordinate time $\tau_{o}$, the region within a proper distance $s \gg \bar{a}$ is completely evacuated. We shall show that the $C$ energy per unit standard length associated with the perturbation must have been

$$
\begin{aligned}
E_{\text {pert }} \equiv \lim _{\rho_{o} \rightarrow \infty}\left\{E\left(\rho_{o}\right)-(1 / G) \ln \rho_{o}\right\}_{\tau=\tau_{o}} & \\
& \geq(1 / G) \ln (s / \bar{a}) .
\end{aligned}
$$

Let $\rho_{s}$ be the radial coordinate at proper distance $s$ from the symmetry axis, and let $\psi\left(\rho_{s}, \tau_{o}\right) \equiv \psi_{s}$. Let $\rho_{o} \gg 1$ be fixed at a point which, at time $\tau_{o}$, has not yet received word of the perturbation. Finally, define $A_{3}\left(\rho_{s}, \tau_{o}\right)=0$, so that (conservation of magnetic flux) $A_{3}\left(\rho_{o}, \tau_{o}\right)=-1$. Thus, $A_{3}$ and $\psi$ have the boundary values

$$
\begin{aligned}
A_{3}\left(\rho_{s}, \tau_{o}\right) & =0, \quad A_{3}\left(\rho_{o}, \tau_{o}\right)=-1, \\
\psi\left(\rho_{s}, \tau_{o}\right) & =\psi_{s}, \quad \psi\left(\rho_{o}, \tau_{o}\right)=\ln \left(1+\rho_{o}^{2}\right)=2 \ln \rho_{o} .
\end{aligned}
$$

According to Eqs. (15) and (16), at time $\tau_{o}$

$$
E\left(\rho_{o}\right) \geq(1 / 4 G) \int_{0}^{\rho_{o}}\left\{\rho \psi^{\prime 2}+\left(e^{2 \psi} / \rho\right) A_{3}^{\prime 2}\right\} d \rho .
$$

But the integral in Eq. (A11) is absolutely minimized with respect to the constraints (A10) by that unique static solution of Eqs. (12a) and (12c) with $A_{2}=0$ which satisfies the constraints. ( $C$-energy minimum principle-cf. the Appendix of Ref. 7.) One can show, by using the techniques of the Appendix of Ref. 7, that this minimum value is given by

$$
\begin{aligned}
E\left(\rho_{o}\right) \geq(1 / 4 G)\left\{2 \ln \left[1+e^{-2\left(b \sigma_{o}+c\right)}\right]\right. \\
-2 \ln \left[1+e^{-2\left(b \sigma_{s}+c\right)}\right]+(1+b)^{2}\left(\sigma_{o}-\sigma_{s}\right),
\end{aligned}
$$

where

$$
\begin{aligned}
& \sigma_{o}=\ln \rho_{o}, \quad \sigma_{s}=\ln \rho_{s}, \quad y_{o}=\rho_{o}^{-1}, \quad y_{s}=\rho_{s} e^{-\psi_{s}}, \\
& b=\left(\sigma_{o}-\sigma_{s}\right)^{-1}(\operatorname{arctanh}[f / a] \\
& -\operatorname{arctanh}[(1+f) / a]\} \\
& c=\left(\sigma_{o}-\sigma_{s}\right)^{-1}\left\{\sigma_{s} \operatorname{arctanh}[(1+f) / a]\right. \\
& \left.-\sigma_{o} \operatorname{arctanh}[f / a]\right\} \text {, } \\
& f=\frac{1}{2}\left[y_{s}{ }^{2}-y_{o}{ }^{2}-1\right] \text {, } \\
& a=\frac{1}{2}\left[4 y_{s}^{2}+\left(y_{s}^{2}-y_{o}^{2}-1\right)^{2}\right]^{1 / 2} \text {. }
\end{aligned}
$$

Because spacetime is flat inside $\rho_{s}$,

$$
y_{s}=\rho_{s} e^{-\psi_{s}}=s / \bar{a} \text {. }
$$

Using this, and expanding Eq. (A12) to lowest order in $\bar{a} / s$ and in $1 / \rho_{o}$, we obtain

$$
E\left(\rho_{o}\right) \geq(1 / 4 G)\left[4 \ln \rho_{o}+4 \ln (s / \bar{a})\right],
$$

which implies Eq. (A9).

Q.E.D.

\section{APPENDIX B : NUMERICAL SOLUTION OF EINSTEIN-MAXWELL EQUATIONS}

The dynamical behavior of Melvin's magnetic universe, when subjected to large perturbations of the form (17), was calculated by numerical integration of the Einstein-Maxwell field equations (12). The calculations were restricted to initial conditions for which $A_{2}=\dot{A}_{2}=0 \quad\left(B_{\varphi}=E_{\zeta}=0\right)$, because this guaranteed that $A_{2}=0$ at all points in space-time [cf. Eqs. (12)], thereby simplifying the computations. There is no reason to believe that qualitatively different behavior would have resulted had this restriction not been imposed.

The integration was performed as follows: A rectangular grid was set up in the $(\rho, \tau)$ plane with spacings $\Delta \rho$ and $\Delta \tau$ between grid points. At a given stage in the computation $\psi, A_{3}$, and $\gamma$ were known at all grid points with $\tau \leq k \Delta \tau$; and one calculated $\psi, A_{3}$, and $\gamma$ at grid points on the "future" hypersurface $\tau=(k+1) \Delta \tau$ from their values on the preceding hypersurfaces $\tau=k \Delta \tau$, $\tau=(k-1) \Delta \tau$, and $\tau=(k-2) \Delta \tau$. This calculation was performed using finite difference approximations to Eqs. (12a), (12c), and (12e). The difference equations were so constructed that they were linear in the unknowns; this was done by ensuring that only the left sides of Eqs. (12a), (12c), and (12e) involved fields evaluated on the "future" hypersurface. On the left 
sides of Eqs. (12a) and (12c), in addition to time derivatives which are quite naturally written so as to involve "future" points, there are the terms $(1 / \rho)\left(\rho \psi^{\prime}\right)$ ' and $\rho\left(A_{3}^{\prime} / \rho\right)$ ' which one would normally evaluate on the "present" hypersurface $\tau=k \Delta \tau$. However, in order to optimize the stability of the difference equations, these expressions were replaced by suitable averages of their values on the present and future hypersurfaces. ${ }^{29}$

The computation was performed in the region $\tau \geq 0$, $0 \leq \rho \leq R+\tau$. [ $R$ is the quantity appearing in Eq. (17). $]$ Boundary conditions of smoothness were imposed at $\rho=0$ (i.e., $\psi^{\prime}, A_{3}{ }^{\prime}$, and $\gamma^{\prime}$ were required to vanish there). At the boundary $\rho=R+\tau, \psi$ and $A_{3}$ were required to

${ }^{29}$ This is a form of the "implicit" method for ensuring stability of difference equations. See, e.g., L. Lapidus, Digital Computation for Chemical Engineers (McGraw-Hill Book Company, Inc., New York, 1962) join on smoothly to Melvin's unperturbed configuration (13). Initial values of $\psi, A_{3}$, and $\gamma$ were introduced on the hypersurfaces $\tau=-\Delta \tau, \tau=0$, and $\tau=+\Delta \tau$ for $0 \leq \rho \leq R$. These initial values were obtained by specifying $\psi$ and $A_{3}$ arbitrarily [except for conditions (17)] at $\tau=0$, setting $\dot{\psi}=\dot{A_{3}}=\dot{\gamma}=0$ there (time-symmetric initial conditions), and then solving equations (12a), (12c), and (12e) to first order in $\Delta \tau$ and $\Delta \rho$, for $\gamma$ at $\tau=0$ and for $\psi, A_{3}$, and $\gamma$ at $\tau= \pm \Delta \tau$.

Once the initial value equations had been solved, the computer attacked the main problem of calculating the fields on successive hypersurfaces $\tau=2 \Delta \tau, \tau=3 \Delta \tau, \cdots$. As it went along, it checked its own calculations at each grid point by comparing $\gamma^{\prime}$ with the right side of Eq. (12d). If they differed by more than ten percent (cumulative error) at any grid point, all calculations to the future of that grid point were considered invalid.

\title{
Fresnel's Equation of Wave Normals for Vacuum Polarization. Application to the Birefringence of the Vacuum
}

\author{
Hugo F. KREMER \\ Instituto de Física da Universidade do Paraná, Paraná, Brazil \\ (Received 30 November 1964; revised manuscript received 5 March 1965)
}

\begin{abstract}
Semiclassical methods are used to study vacuum polarization. Using the modified Lagrangian, an equation analogous to Fresnel's equation of wave normals is derived and applied to the study of the birefringence of the vacuum.
\end{abstract}

$\mathbf{I}^{\mathrm{T}}$ is well known that quantum electrodynamics predicts the existence of nonlinear effects between electromagnetic fields in the vacuum..$^{1-3}$ The creation of virtual electron-positron pairs results in the interaction of two photons and leads to additional terms in the Lagrangian, which are quadratic in the invariants

$$
\begin{gathered}
F=\frac{1}{2} f_{\alpha \beta} f^{\alpha \beta}=\mathbf{B}^{2}-\mathbf{E}^{2} ; \quad G=\frac{1}{4} f_{\alpha \beta} f^{* \alpha \beta}=\mathbf{B} \cdot \mathbf{E} ; \\
f^{* \alpha \beta}=\frac{1}{2} \epsilon^{\alpha \beta \rho \sigma} f_{\rho \sigma} .
\end{gathered}
$$

According to Karplus and Neuman, ${ }^{4}$ and Schwinger, ${ }^{5}$ the additional terms are given up to the order $\alpha^{2}$ by

$$
L_{\mathrm{int}}=-\left(2 \alpha^{2} / 45 m^{4}\right)\left(F^{2}+7 G^{2}\right),
$$

where we adopt rationalized units and $\hbar=c=1 ; \alpha$ is the fine-structure constant, and $m$ is the mass of the electron.

Recently, semiclassical methods have been used to

${ }^{1}$ H. Euler, Ann. Physik 26, 398 (1936).

${ }^{2}$ W. Heisenberg and H. Euler, Z. Physik 98, 714 (1936).

3 V. S. Weisskopf, Kgl. Danske Videnskab. Selskab, Mat. Fys. Medd. 16, No. 6 (1936).

${ }_{4}^{4}$ R. Karplus and M. Neuman, Phys. Rev. 80, 380 (1950); 83, 776 (1951).

${ }_{5}^{5}$ J. Schwinger, Phys. Rev. 82, 664 (1951). study the nonlinear interaction of light in a vacuum. ${ }^{6-7}$ However, the use of Fresnel's equation for crystal optics in the study of the birefringence of the vacuum is not strictly valid. It is our purpose to review this question by deriving a Fresnel equation valid for polarizable vacuum and using it to study the process suggested by Klein and Nigam. We show that the birefringence is very peculiar, presenting two extraordinary waves at right angles to the applied uniform static field $E_{s}$, both with phase velocities smaller than $c$.

\section{FRESNEL'S EQUATION FOR VACUUM POLARIZATION}

We start from an arbitrary Lagrangian $L=L(F, G)$, a function of the two invariants $F$ and $G$ constructed from the electromagnetic field $f_{\alpha \beta}$. The electromagnetic induction $p_{\alpha \beta}$ is defined $\mathrm{by}^{8}$

$$
p^{\alpha \beta} \equiv \partial L / \partial f_{\alpha \beta}=2 L_{F} f^{\alpha \beta}+L_{G} f^{* \alpha \beta},
$$

where $L_{F}=\partial L / \partial F, L_{G}=\partial L / \partial G$.

${ }^{6} \mathrm{~J}$. McKenna and P. M. Platzman, Phys. Rev. 129, 2354 (1963).

${ }^{7}$ J. J. Klein and B. P. Nigam, Phys. Rev. 135, B1279 (1964).

${ }^{8} \mathrm{See}$, for example, M. Born and L. Infeld, Proc. Roy. Soc. (London) A144, 425 (1934). 